\title{
The Methods of the Synthesis of the Nonlinear Regulators for the Distributed One-Dimension Control Objects
}

\author{
Yury Volerevich Ilyushin ${ }^{1}$, Dmitry Anatolyevich Pervukhin ${ }^{1}$, Olga Vladimirovna Afanasieva ${ }^{1}$, Mikhail \\ Petrovich Afanasyev ${ }^{1} \&$ Sergey Viktorovich Kolesnichenko ${ }^{1}$ \\ ${ }^{1}$ National Mineral Resources Universiti (University of Mines), Russian Federation \\ Correspondence: Yury Volerevich Ilyushin, National Mineral Resources Universiti (University of Mines), \\ Russian Federation. E-mail: bdbyu@rambler.ru
}

Received: October 15, 2014

Accepted: October 25, $2014 \quad$ Online Published: December 7, 2014

doi:10.5539/mas.v9n2p42

URL: http://dx.doi.org/10.5539/mas.v9n2p42

\begin{abstract}
In this paper the method of synthesis of distributed regulator, uniform control object is regarded, which is based on the specified inaccuracy. The function of the initial heating has been received; the mathematical modeling of the process has been conducted as well as the analysis of the results. With the help of the obtained regulator hardware and software complex has been designed in the Pascal programming language, the complex allows to simulate the behavior of the temperature fields in the isotropic rod. The article presents the simulation of the temperature process for different configurations of the system. Namely, there is simulation with different number of impulsive heating sources with the relay control principle. The practical results of these studies suggest the possibility of building silicon carbide heating element, made in the form of an isotropic rod.
\end{abstract}

Keywords: Green's function, thermal field, discretization interval, management object, analysis, synthesis

\section{Introduction.}

Electric tunnel kiln of conveyor type has a number of advantages and some disadvantages. One of the major disadvantages of electric furnaces of this type is that they are expensive to maintain the value of energy resources, so for heating of one of the heating elements the energy, equal to $0.12 \%$ of the value of one item is consumed. In the tunnel kiln hexagonal silicon carbide rods are arranged along the entire length of the firing chamber.

Such long heating elements require a long time to worming up and a lot of energy demands, which in its turn has a significant impact on the final cost of the product. Let's consider the possibility of the cost reduction at the expense of the use of impulsive heating elements, which in their turn will heat the silicon carbide rods for maintaining a predetermined temperature regime of the kiln section. At the expense of the short-term switching on, the energy saving and, as a consequence, the reduction of the cost of the product will occur.

\section{The Task Assignment}

In modal image analysis solutions are infinite or finite linear combinations of "modes", as a combination of exponential-periodic basis functions of time and coordinates (Tikhonov, \& Samara, 1965).

On the basis of process models program controls' synthesis is implemented, by the formalizing of the requirements into single-point or multi-point target conditions (equations, inequations), which are represented by the requirements of minimizing of quality functionals. Within the correlations between the coordinates (factors) the analytical solutions of the heat conduction operators are used or difference schemes for the corresponded tasks. This allows you to purchase a great variety of algorithms for software control. These algorithms, with the associated generalization can be designed to create a system of locally-optimal or locally-oriented management.

Let's us set the problem of stabilization of the temperature field in a certain temperature range. Approaches to the synthesis of stabilizing controls may be considered on the basis of variant target conditions and solution of mathematical programming problems by numerical methods. As a result, the solutions of target conditions it is required to obtain an explicit representation of the laws of feedbacks, which in its turn allows us to investigate the conditions of stability of closed systems of stabilization of distributed objects. In the case of $T(x, t)$ is analytical solution of initial-boundary task, the system of the targeted equations has a form of:

$$
T(x, t)=T\left(x, T_{\mathrm{det}, i}\right) \text {; }
$$


where $T\left(x, T_{d e t, i}\right)$ - if an analytical solution is determined up to the parameters of the heated body and external disturbances, the system can be solved relating to the last variables.

\section{Methods}

Target conditions of inequation type, determine interval requirements for object coordinates for a total of predetermined moments of time. The problem is reduced to the solution of a finite number of systems of inequations on specified relating to the parameters of input effects or object parameters:

$$
T \leq T(x, t)=T\left(x, t_{\mathrm{det}, i}\right) \leq T^{+} .
$$

Target conditions in the form of minimizing the functional, which set on deviations of temperature regimes (from specified requirements) is realized by minimizing the object's parameters or input actions, which will reduce the problem of synthesis to problems of finite-dimensional minimization:

$$
J\left|T\left(x_{m}, t_{n}\right), T\left(x_{\mathrm{det}, m}, t_{\mathrm{det}, i}\right)\right| \rightarrow \min :
$$

where $T\left(x_{m}, t_{n}\right), u T\left(x_{\mathrm{det}, m}, t_{\mathrm{det}, i}\right)$ analytical temperature values at predetermined time points and the coordinates as well as their numerical values. Next, on the described mathematical model we will conduct synthesis of control effects on the rod (Pershin, (2007); Ilyushin, (2011); Kolesnikov, (2009)).

$$
\frac{\partial T(x, t)}{\partial t}=a^{2} \frac{\partial^{2} T(x, t)}{\partial x^{2}},
$$

where initial boundary conditions:

$$
T(x, 0)=T_{0}(x) ; T(0, t)=T(l, t)=0 .
$$

It is required to find the parameters of the function $T(x, t)$, solutions, which are selected according to the predetermined initial conditions, which may be equation, inequation, or conditions. Where the synthesis is carried out, using the analytical solutions of initial-boundary tasks. For the presentation of the generalized solution we may use the formal series

$$
T(x, t)=\frac{2}{l} \sum_{n=1}^{\infty} a_{n} \exp \left[-\left(\frac{n \pi a}{l}\right)^{2} t\right] \sin \frac{n \pi}{l} x .
$$

With the use of the single-point conditions by time and coordinates the problem is reduced to the solution of the equation relating to the following parameters:

$$
\frac{2 a_{1}}{l} \exp \left[-\left(\frac{\pi a}{l}\right)^{2} t^{*}\right] \sin \frac{\pi}{l} x^{*}=T_{\mathrm{det}}\left(x^{*}, t^{*}\right) .
$$

This solution is a nonlinear algebraic equation, relating to the parameters that provide the implementation of the target conditions. In other words, this function is a criterion for regulation of the temperature field. In the case of multi-point target conditions, which specify temperature conditions of the flock of points of time of coordinate axes, we can reduce the synthesis problem to the problem of solving a system of equations, relating to the parameters

$$
T(x, t) \approx \frac{2 a_{1}}{l} \exp \left[-\left(\frac{\pi a}{l}\right)^{2} t_{i}\right] \sin \frac{\pi}{l} x^{*}=T_{i, \mathrm{det}}\left(x^{*}, t_{i}\right) .
$$

Using a series with a finite number of summand, the solution of the problem is reduced to the following equation:

$$
T\left(x_{i}, t_{j}\right)=\frac{2}{l} \sum a_{n} \exp \left[-\left(\frac{n \pi a}{l}\right)^{2} t_{j}\right] \sin \frac{n \pi}{l} x_{i}=T_{i, j, 3 a d}\left(x_{i}, t_{j}\right) .
$$

Let's perform the task of stabilization of the temperature field in the rod at a value of $T_{\text {det }}=$ const, using the obtained function. At the initial time $\tau_{0}=0$ the switching on of all the sources of temperature will occur. Each temperature source affects the neighboring sensors and temperature fields, caused by nearby thermal sources. Applying the Green's function, we obtain the following expression: the effect of the first source on the seven following sensors

$$
T\left(x_{1}, t, \tau_{0}, \xi_{1}\right)=\frac{2}{l} \sum_{n=1}^{k} \exp \left[-\left(\frac{\pi n a}{l}\right)^{2} t\right] \sin \frac{\pi n}{l} x_{1} \sin \frac{\pi n}{l} \xi_{1}
$$




$$
\begin{aligned}
& T\left(x_{1}, t, \tau_{0}, \xi_{2}\right)=\frac{2}{l} \sum_{n=1}^{k} \exp \left[-\left(\frac{\pi n a}{l}\right)^{2} t\right] \sin \frac{\pi n}{l} x_{1} \sin \frac{\pi n}{l} \xi_{2} \\
& T\left(x_{1}, t, \tau_{0}, \xi_{3}\right)=\frac{2}{l} \sum_{n=1}^{k} \exp \left[-\left(\frac{\pi n a}{l}\right)^{2} t\right] \sin \frac{\pi n}{l} x_{1} \sin \frac{\pi n}{l} \xi_{3} \\
& T\left(x_{1}, t, \tau_{0}, \xi_{4}\right)=\frac{2}{l} \sum_{n=1}^{k} \exp \left[-\left(\frac{\pi n a}{l}\right)^{2} t\right] \sin \frac{\pi n}{l} x_{1} \sin \frac{\pi n}{l} \xi_{4}
\end{aligned}
$$

The effect of all sources on the sensors $x_{1}, x_{2}, x_{3}$ :

$$
\begin{aligned}
& T\left(x_{1}, t, \tau_{0}\right)=\sum_{i=1}^{3} \sum_{n=1}^{k} \frac{2}{l} \exp \left[-\left(\frac{\pi n a}{l}\right)^{2} t\right] \sin \frac{\pi n}{l} x_{1} \sin \frac{\pi n}{l} \xi_{i} ; \\
& T\left(x_{2}, t, \tau_{0}\right)=\sum_{i=1}^{3} \sum_{n=1}^{k} \frac{2}{l} \exp \left[-\left(\frac{\pi n a}{l}\right)^{2} t\right] \sin \frac{\pi n}{l} x_{2} \sin \frac{\pi n}{l} \xi_{i} ; \\
& T\left(x_{3}, t, \tau_{0}\right)=\sum_{i=1}^{3} \sum_{n=1}^{k} \frac{2}{l} \exp \left[-\left(\frac{\pi n a}{l}\right)^{2} t\right] \sin \frac{\pi n}{l} x_{3} \sin \frac{\pi n}{l} \xi_{i} ;
\end{aligned}
$$

In general terms, for the $N$ number of sources and sensors $d$, expression of the function of the initial warming at the observation point $x j$ will be as follows

$$
T\left(x_{j}, t, \tau_{0}\right)=\sum_{i=1}^{d} \sum_{n=1}^{k} \frac{2}{l} \exp \left[-\left(\frac{\pi n a}{l}\right)^{2} t\right] \sin \frac{\pi n}{l} x_{j} \sin \frac{\pi n}{l} \xi_{i}
$$

where $j=1,2, \ldots d$.

After some time, the temperature of the rod will decrease due to the zero-point boundary conditions. At the point of the isotropic rod $x_{j}$ the function, $T\left(x_{j}, t, \tau_{0}\right)$ decreasing, will achieve the predetermined value $T_{\text {det. }}$ Let us say, with $t=\tau_{1}$, the impulsive source with the relay control is switched on $\xi_{\mathrm{j}}$, which corresponds to $x_{\mathrm{j}}$. Then for instance, at the $\tau_{1}$ point of time the $x_{1}$ the temperature source outputs the value equal to the predetermined temperature regime $T_{\text {det }}$. Next, the $\xi_{1}$ source is switch on and effects on all the sensors.

$$
\begin{aligned}
& T\left(x_{1}, t, \tau_{1}, \xi_{1}\right)=\sum_{n=1}^{k} \frac{2}{l} \exp \left[-\left(\frac{\pi n a}{l}\right)^{2}\left(t-\tau_{1}\right)\right] \sin \frac{\pi n}{l} x_{1} \sin \frac{\pi n}{l} \xi_{1} ; \\
& T\left(x_{2}, t, \tau_{1}, \xi_{1}\right)=\sum_{n=1}^{k} \frac{2}{l} \exp \left[-\left(\frac{\pi n a}{l}\right)^{2}\left(t-\tau_{1}\right)\right] \sin \frac{\pi n}{l} x_{2} \sin \frac{\pi n}{l} \xi_{1} ; \\
& T\left(x_{3}, t, \tau_{1}, \xi_{1}\right)=\sum_{n=1}^{k} \frac{2}{l} \exp \left[-\left(\frac{\pi n a}{l}\right)^{2}\left(t-\tau_{1}\right)\right] \sin \frac{\pi n}{l} x_{3} \sin \frac{\pi n}{l} \xi_{1} ;
\end{aligned}
$$

The function of the initial heating continues to have residual effects on each temperature sensor. Let's calculate the total thermal effect of all the sensors on all sources:

$$
T\left(x_{1}, t\right)=\sum_{i=1}^{3} \sum_{n=1}^{k} \frac{2}{l} \exp \left[-\left(\frac{\pi n a}{l}\right)^{2} t\right] \sin \frac{\pi n}{l} x_{1} \sin \frac{\pi n}{l} \xi_{i}+\sum_{n=1}^{k} \frac{2}{l} \exp \left[-\left(\frac{\pi n a}{l}\right)^{2}\left(t-\tau_{1}\right)\right] \sin \frac{\pi n}{l} x_{1} \sin \frac{\pi n}{l} \xi_{1} ;
$$




$$
\begin{aligned}
& T\left(x_{2}, t\right)=\sum_{i=1}^{3} \sum_{n=1}^{k} \frac{2}{l} \exp \left[-\left(\frac{\pi n a}{l}\right)^{2} t\right] \sin \frac{\pi n}{l} x_{2} \sin \frac{\pi n}{l} \xi_{i}+\sum_{n=1}^{k} \frac{2}{l} \exp \left[-\left(\frac{\pi n a}{l}\right)^{2}\left(t-\tau_{1}\right)\right] \sin \frac{\pi n}{l} x_{2} \sin \frac{\pi n}{l} \xi_{1} \\
& T\left(x_{3}, t\right)=\sum_{i=1}^{3} \sum_{n=1}^{k} \frac{2}{l} \exp \left[-\left(\frac{\pi n a}{l}\right)^{2} t\right] \sin \frac{\pi n}{l} x_{3} \sin \frac{\pi n}{l} \xi_{i}+\sum_{n=1}^{k} \frac{2}{l} \exp \left[-\left(\frac{\pi n a}{l}\right)^{2}\left(t-\tau_{1}\right)\right] \sin \frac{\pi n}{l} x_{3} \sin \frac{\pi n}{l} \xi_{1}
\end{aligned}
$$

In more simplified form, we obtain the following results for the first three points:

$$
T\left(x_{1}, t\right)=T\left(x_{1}, t, \tau_{0}\right)+T\left(x_{1}, t, \tau_{1}\right), T\left(x_{2}, t\right)=T\left(x_{2}, t, \tau_{0}\right)+T\left(x_{2}, t, \tau_{1}\right), T\left(x_{1}, t\right)=T\left(x_{1}, t, \tau_{0}\right)+T\left(x_{1}, t, \tau_{1}\right) .
$$

With the course of time $\tau_{2}$ in the point $x_{3}$ the function, decreasing, achieved the value of the predetermined temperature regime $T_{\text {det. }}$. After that the impulsive relay source $\zeta_{3}$ is switch on, which is located with the correspondence to the $x_{3}$ sensor and creates temperature effect on all points of the rod. At each point of the rod the impact of the function of the initial heating extends. Based on the above stated we obtain:

$$
\begin{gathered}
T\left(x_{1}, t\right)=\sum_{i=1}^{3} \sum_{n=1}^{k} \frac{2}{l} \exp \left[-\left(\frac{\pi n a}{l}\right)^{2} t\right] \sin \frac{\pi n}{l} x_{1} \sin \frac{\pi n}{l} \xi_{i}+ \\
\sum_{n=1}^{k} \frac{2}{l} \exp \left[-\left(\frac{\pi n a}{l}\right)^{2}\left(t-\tau_{1}\right)\right] \sin \frac{\pi n}{l} x_{1} \sin \frac{\pi n}{l} \xi_{1}+\sum_{n=1}^{k} \frac{2}{l} \exp \left[-\left(\frac{\pi n a}{l}\right)^{2}\left(t-\tau_{2}\right)\right] \sin \frac{\pi n}{l} x_{1} \sin \frac{\pi n}{l} \xi_{3} \\
T\left(x_{2}, t\right)=\sum_{i=1}^{3} \sum_{n=1}^{k} \frac{2}{l} \exp \left[-\left(\frac{\pi n a}{l}\right)^{2} t\right] \sin \frac{\pi n}{l} x_{2} \sin \frac{\pi n}{l} \xi_{i}+ \\
\sum_{n=1}^{k} \frac{2}{l} \exp \left[-\left(\frac{\pi n a}{l}\right)^{2}\left(t-\tau_{1}\right)\right] \sin \frac{\pi n}{l} x_{2} \sin \frac{\pi n}{l} \xi_{1}+\sum_{n=1}^{k} \frac{2}{l} \exp \left[-\left(\frac{\pi n a}{l}\right)^{2}\left(t-\tau_{2}\right)\right] \sin \frac{\pi n}{l} x_{2} \sin \frac{\pi n}{l} \xi_{3} ; \\
T\left(x_{3}, t\right)=\sum_{i=1}^{3} \sum_{n=1}^{k} \frac{2}{l} \exp \left[-\left(\frac{\pi n a}{l}\right)^{2} t\right] \sin \frac{\pi n}{l} x_{3} \sin \frac{\pi n}{l} \xi_{i}+ \\
\sum_{n=1}^{k} \frac{2}{l} \exp \left[-\left(\frac{\pi n a}{l}\right)^{2}\left(t-\tau_{1}\right)\right] \sin \frac{\pi n}{l} x_{3} \sin \frac{\pi n}{l} \xi_{1}+\sum_{n=1}^{k} \frac{2}{l} \exp \left[-\left(\frac{\pi n a}{l}\right)^{2}\left(t-\tau_{2}\right)\right] \sin \frac{\pi n}{l} x_{3} \sin \frac{\pi n}{l} \xi_{3}
\end{gathered}
$$

Then we will receive the following temperature fields' correlations:

$$
\begin{gathered}
T\left(x_{1}, t\right)=T\left(x_{1}, t, \tau_{0}\right)+T\left(x_{1}, t, \tau_{1}\right)+T\left(x_{1}, t, \tau_{2}\right)+\ldots+T\left(x_{1}, t, \tau_{p}\right) ; T\left(x_{2}, t\right)=T\left(x_{2}, t, \tau_{0}\right)+T\left(x_{2}, t, \tau_{1}\right)+ \\
T\left(x_{2}, t, \tau_{2}\right)+\ldots+T\left(x_{2}, t, \tau_{p}\right) ; \\
T\left(x_{3}, t\right)=T\left(x_{3}, t, \tau_{0}\right)+T\left(x_{3}, t, \tau_{1}\right)+T\left(x_{3}, t, \tau_{2}\right)+\ldots+T\left(x_{3}, t, \tau_{p}\right) ;
\end{gathered}
$$

The effect of all functions on all sources may be expressed by the following equation

$$
\begin{aligned}
& T(x, t)=\sum_{i=1}^{d} \sum_{n=1}^{k} \frac{2}{l} \exp \left[-\left(\frac{\pi n a}{l}\right)^{2} t\right] \sin \frac{\pi n}{l} x \sin \frac{\pi n}{l} \xi_{i}=\frac{2}{l}\left[\left(\exp \left[-\left(\frac{\pi a}{l}\right)^{2} t\right] \sin \frac{\pi}{l} x \sin \frac{\pi}{l} \xi_{1}+\right.\right. \\
&+\left.\exp \left[-\left(\frac{2 \pi a}{l}\right)^{2} t\right] \sin \frac{2 \pi}{l} x \sin \frac{2 \pi}{l} \xi_{1}+\ldots+\exp \left[-\left(\frac{k \pi a}{l}\right)^{2} t\right] \sin \frac{k \pi}{l} x \sin \frac{k \pi}{l} \xi_{1}\right)+ \\
&+\left(\exp \left[-\left(\frac{\pi a}{l}\right)^{2} t\right] \sin \frac{\pi}{l} x \sin \frac{\pi}{l} \xi_{2}+\exp \left[-\left(\frac{2 \pi a}{l}\right)^{2} t\right] \sin \frac{2 \pi}{l} x \sin \frac{2 \pi}{l} \xi_{2}+\ldots+\right. \\
&\left.+\exp \left[-\left(\frac{k \pi a}{l}\right)^{2} t\right] \sin \frac{k \pi}{l} x \sin \frac{k \pi}{l} \xi_{2}\right)+\left(\exp \left[-\left(\frac{\pi a}{l}\right)^{2} t\right] \sin \frac{\pi}{l} x \sin \frac{\pi}{l} \xi_{3}+\right. \\
&\left.\left.+\exp \left[-\left(\frac{2 \pi a}{l}\right)^{2} t\right] \sin \frac{2 \pi}{l} x \sin \frac{2 \pi}{l} \xi_{3}+\ldots+\exp \left[-\left(\frac{k \pi a}{l}\right)^{2} t\right] \sin \frac{k \pi}{l} x \sin \frac{k \pi}{l} \xi_{3}\right)+\ldots\right]=
\end{aligned}
$$




$$
\begin{aligned}
& =\frac{2}{l}\left[\exp \left[-\left(\frac{\pi a}{l}\right)^{2} t\right] \sin \frac{\pi}{l} x\left(\sin \frac{\pi}{l} \xi_{1}+\sin \frac{\pi}{l} \xi_{2}+\sin \frac{\pi}{l} \xi_{3}+\ldots\right)+\right. \\
& +\exp \left[-\left(\frac{2 \pi a}{l}\right)^{2} t\right] \sin \frac{2 \pi}{l} x\left(\sin \frac{2 \pi}{l} \xi_{1}+\sin \frac{2 \pi}{l} \xi_{2}+\sin \frac{2 \pi}{l} \xi_{3}+\ldots\right)+ \\
+ & \left.\exp \left[-\left(\frac{3 \pi a}{l}\right)^{2} t\right] \sin \frac{3 \pi}{l} x\left(\sin \frac{3 \pi}{l} \xi_{1}+\sin \frac{3 \pi}{l} \xi_{2}+\sin \frac{3 \pi}{l} \xi_{3}+\ldots\right)+\ldots\right]
\end{aligned}
$$

Or in simplified form

$$
\begin{gathered}
T(x, t)=\frac{2}{l}\left[\exp \left[-\left(\frac{\pi a}{l}\right)^{2} t\right] \sin \frac{\pi}{l} x \sum_{i=1}^{d} \sin \frac{\pi}{l} \xi_{i}+\exp \left[-\left(\frac{3 \pi a}{l}\right)^{2} t\right] \sin \frac{3 \pi}{l} x \sum_{i=1}^{d / 3} \sin \frac{3 \pi}{l} \xi_{i}+\right. \\
\left.+\exp \left[-\left(\frac{5 \pi a}{l}\right)^{2} t\right] \sin \frac{5 \pi}{l} x \sum_{i=1}^{d / 5} \sin \frac{5 \pi}{l} \xi_{i}+\ldots\right]
\end{gathered}
$$

At the initial point of time (at $t=0$ ), the expression has form of:

$$
T(x, 0)=\frac{2}{l}\left[\sin \frac{\pi}{l} x \sum_{i=1}^{d} \sin \frac{\pi}{l} \xi_{i}+\sin \frac{3 \pi}{l} x \sum_{i=1}^{d / 3} \sin \frac{3 \pi}{l} \xi_{i}+\sin \frac{5 \pi}{l} x \sum_{i=1}^{d / 5} \sin \frac{5 \pi}{l} \xi_{i}+\ldots\right] ;
$$

Where the power of the initial signal will depend on the number of constituents of the Fourier's series

$$
\begin{gathered}
S_{1}=\int_{0}^{l} \sin \frac{\pi}{l} x d x=-\left.\frac{l}{\pi} \cos \frac{\pi}{l} x\right|_{0} ^{l}=-\frac{l}{\pi} \cos \pi+\frac{l}{\pi} \cos 0=\frac{2 l}{\pi} \\
S_{2}=\int_{0}^{l / 3} \sin \frac{3 \pi}{l} x d x=-\left.\frac{l}{3 \pi} \cos \frac{3 \pi}{l} x\right|_{0} ^{\frac{l}{3}}=-\frac{l}{3 \pi} \cos \pi+\frac{l}{3 \pi} \cos 0=\frac{2 l}{3 \pi}=\frac{1}{3}\left(\frac{2 l}{\pi}\right) ; \\
S_{3}=\int_{0}^{l / 5} \sin \frac{5 \pi}{l} x d x=-\left.\frac{l}{5 \pi} \cos \frac{5 \pi}{l} x\right|_{0} ^{l / 5}=-\frac{l}{5 \pi} \cos \pi+\frac{l}{5 \pi} \cos 0=\frac{2 l}{5 \pi}=\frac{1}{5}\left(\frac{2 l}{\pi}\right) ; \\
S_{n}=\int_{0}^{l / n} \sin \frac{\pi n}{l} x d x=\frac{1}{n}\left(\frac{2 l}{\pi}\right), \text { where } n-\text { odd. }
\end{gathered}
$$

So far as $S_{2}=\frac{1}{3} S_{1} ; \quad S_{3}=\frac{1}{5} S_{1} ; \quad \ldots . ; S_{n}=\frac{1}{n} S_{1}$, than

$$
\begin{gathered}
T\left(\frac{l}{2} ; 0\right)=\frac{2}{l}\left[\sum_{i=1}^{d} \sin \frac{\pi}{l} \xi_{i}-\sum_{i=1}^{d / 3} \sin \frac{3 \pi}{l} \xi_{i}+\sum_{i=1}^{d / 5} \sin \frac{5 \pi}{l} \xi_{i}-\ldots\right]= \\
=\frac{2}{l}\left[\sum_{i=1}^{d} \sin \frac{\pi}{l} \xi_{i}-\frac{1}{3} \sum_{i=1}^{d} \sin \frac{\pi}{l} \xi_{i}+\frac{1}{5} \sum_{i=1}^{d} \sin \frac{\pi}{l} \xi_{i}-\ldots\right]=\frac{2}{l}\left(\sum_{i=1}^{d} \sin \frac{\pi}{l} \xi_{i}\right)\left(1-\frac{1}{3}+\frac{1}{5}-\frac{1}{7}+\frac{1}{9}-\ldots\right)= \\
=\frac{2}{l} \sum_{n=1}^{\infty} \frac{(-1)^{n-1}}{2 n-1} \sum_{i=1}^{d} \sin \frac{\pi}{l} \xi_{i} ;
\end{gathered}
$$


As $x=\frac{l}{4}: \sin \frac{\pi}{l} x=\frac{\sqrt{2}}{2}, \sin \frac{3 \pi}{l} x=\frac{\sqrt{2}}{2}, \sin \frac{5 \pi}{l} x=-\frac{\sqrt{2}}{2}, \ldots$, then

$$
\begin{gathered}
T\left(\frac{l}{4} ; 0\right)=\frac{2}{l}\left(\sum_{i=1}^{d} \sin \frac{\pi}{l} \xi_{i}\right)\left(\frac{\sqrt{2}}{2}+\frac{1}{3} \frac{\sqrt{2}}{2}-\frac{1}{5} \frac{\sqrt{2}}{2}-\frac{1}{7} \frac{\sqrt{2}}{2}+\ldots\right)==\frac{\sqrt{2}}{l}\left(\sum_{i=1}^{d} \sin \frac{\pi}{l} \xi_{i}\right)\left(1+\frac{1}{3}-\frac{1}{5}-\frac{1}{7}+\frac{1}{9}+\ldots\right) ; \\
\text { As } x=\frac{l}{6}: \sin \frac{\pi}{l} x=\frac{1}{2}, \sin \frac{3 \pi}{l} x=1, \sin \frac{5 \pi}{l} x=\frac{1}{2}, \sin \frac{7 \pi}{l} x=-\frac{1}{2}, \sin \frac{9 \pi}{l} x=-1, \ldots, \text { then } \\
T\left(\frac{l}{6} ; 0\right)=\frac{1}{l}\left(\sum_{i=1}^{l} \sin \frac{\pi}{l} \xi_{i}\right)\left(1+\frac{2}{3}+\frac{1}{5}-\frac{1}{7}-\frac{2}{9}+\ldots\right)
\end{gathered}
$$

At any fixed point of section:

$$
\begin{aligned}
& T\left(x_{j}, t\right)=\sum_{i=1}^{d} \sum_{n=1}^{k} \frac{2}{l} \exp \left[-\left(\frac{\pi n a}{l}\right)^{2} t\right] \sin \frac{\pi n}{l} x_{j} \sin \frac{\pi n}{l} \xi_{i}+ \\
& +\sum_{p} \sum_{n=1}^{k} \frac{2}{l} \exp \left[-\left(\frac{\pi n a}{l}\right)^{2}\left(t-\tau_{p}\right)\right] \sin \frac{\pi n}{l} x_{j} \sin \frac{\pi n}{l} \xi_{z(p)} ;
\end{aligned}
$$

\section{Results}

The function, which takes into account the behavior of the temperature field in the course of time, makes it possible to consider a distributed control object as a unified system of thermal fields. Let's consider the interaction of thermal fields in the rod of $l$ length, and of the type of material, which is characterized by the $\mathrm{a}^{2}$ thermal conductance. Let us assume that the rod is negligibly thin, that is, we assume it to be one-dimensional (Kolesnikov, et al. (2007); Kolesnikov, et al. (2007); Zarembo, \& Kolesnikov (2006); Zarembo, \& Kolesnikov (2006)). Let us solve the problem of the control system synthesis, the stabilization of the temperature field. To do this, let us implement the Green's function in the Delphi language and write a program that simulates the behavior of the temperature field in the course of time. The program code that implements this task is as follows:

Begin

$\mathrm{g}[\mathrm{j}]:=0$;

for $\mathrm{i}:=1$ to $\mathrm{d}$ do

Begin

For $\mathrm{n}:=1$ to $\mathrm{k}$ do

Begin

$$
\text { vspom: }=(2 * \exp (((-\mathrm{pi} * \mathrm{n} * \mathrm{a}) / \mathrm{l}) *((\mathrm{pi} * \mathrm{n} * \mathrm{a}) / \mathrm{l}) * \mathrm{t} 1)) * \sin \left(\mathrm{pi}{ }^{*} \mathrm{n} * \mathrm{x}[\mathrm{j}] / 1\right) * * \sin (\mathrm{pi} * \mathrm{n} * \mathrm{ksi}[\mathrm{i}] / \mathrm{l}) / \mathrm{l}
$$

The code of the placement of temperature sensors has the form of:

if $(\mathrm{i}<\mathrm{d}+1)$ then

begin

$$
\begin{aligned}
& \mathrm{x}[\mathrm{i}]:=\mathrm{i}^{*}(1 / \mathrm{d}) ; \\
& \mathrm{ksi}[\mathrm{i}]:=\mathrm{x}[\mathrm{i}]
\end{aligned}
$$

end;

for $\mathrm{v}:=1$ to $\mathrm{d} d o$

begin

if $\mathrm{x}[\mathrm{v}]<\mathrm{k} 3$ then

begin 


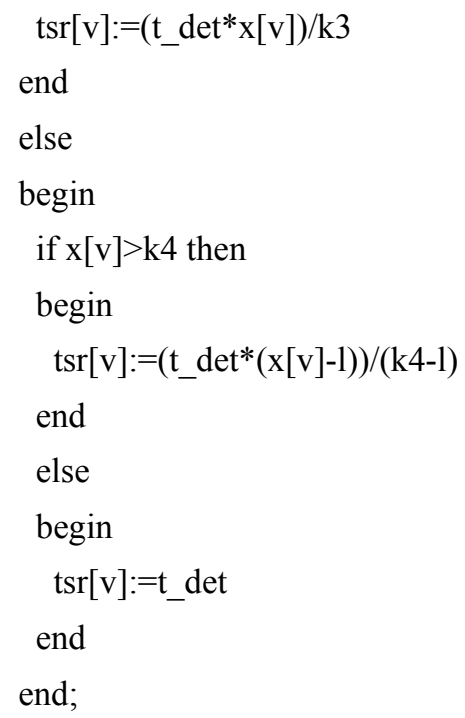

Where $x[i]$ is the temperature sensor and ksi[i] is the heating element. In the program code, the position $x[i]$ is programmed at the same cross-section, where ksi[i] is. This arrangement of heating elements with respect to the corresponding sensors allows fixing the values of the temperature field immediately after the impulse. However, the software complex allows arranging the temperature sensor at some distance from the heat source. This function of software algorithm allows, if it is necessary, measuring the temperature in different parts of the rod.

Let us put a series of experiments on various metals and objects of various lengths. The aim of the study is the identification of dependencies of the material and the method of heating on the number of heating elements and their location. As the initial data for the simulation the following values of the object are taken: $l=10, k=10, d=9$, $x_{1}=\xi_{1}=1, T_{\operatorname{det}}=0.3, \xi \in\{1,2,3,4,5,6,7,8,9\}, a^{2}=0.01$. We obtain the following values of the temperature at different numbers of the heating points.

According to the Table 1 it is clear that in the middle of the rod the temperature is higher than at its ends. This is due to the so-called heat sink. The chart of the values of the temperature field may be seen in Figure 1.

Table 1 . The table calculation results by the number of sensors

\begin{tabular}{|c|c|c|c|c|}
\hline$d=9$ & $d=8$ & $d=7$ & $d=6$ & $d=5$ \\
\hline $\operatorname{tmas}[1.690]=0.19$ & $\operatorname{tmas}[1.690]=0.19$ & $\operatorname{tmas}[1.690]=0.18$ & $\operatorname{tmas}[1.690]=0.18$ & $\operatorname{tmas}[1.690]=0.48$ \\
\hline $\operatorname{tmas}[2.690]=0.37$ & $\operatorname{tmas}[2.690]=0.36$ & $\operatorname{tmas}[2.690]=0.31$ & $\operatorname{tmas}[2.690]=0.32$ & $\operatorname{tmas}[2.690]=0.39$ \\
\hline $\operatorname{tmas}[3.690]=0.49$ & $\operatorname{tmas}[3.690]=0.47$ & $\operatorname{tmas}[3.690]=0.43$ & $\operatorname{tmas}[3.690]=0.37$ & $\operatorname{tmas}[3.690]=0.39$ \\
\hline $\operatorname{tmas}[4.690]=0.56$ & $\operatorname{tmas}[4.690]=0.51$ & $\operatorname{tmas}[4.690]=0.43$ & $\operatorname{tmas}[4.690]=0.32$ & $\operatorname{tmas}[4.690]=0.38$ \\
\hline $\operatorname{tmas}[5.690]=0.56$ & $\operatorname{tmas}[5.690]=0.47$ & $\operatorname{tmas}[5.690]=0.34$ & $\operatorname{tmas}[5.690]=0.18$ & $\operatorname{tmas}[5.690]=0.45$ \\
\hline $\operatorname{tmas}[6.690]=0.49$ & $\operatorname{tmas}[6.690]=0.36$ & $\operatorname{tmas}[6.690]=0.19$ & $\operatorname{tmas}[6.690]=0.26$ & \\
\hline $\operatorname{tmas}[7.690]=0.37$ & $\operatorname{tmas}[7.690]=0.19$ & $\operatorname{tmas}[7.690]=0.42$ & & \\
\hline $\operatorname{tmas}[8.690]=0.19$ & $\operatorname{tmas}[8.690]=0.78$ & & & \\
\hline \multicolumn{5}{|l|}{$\operatorname{tmas}[9.690]=0.14$} \\
\hline$d=14$ & $d=13$ & $d=12$ & $d=11$ & $d=10$ \\
\hline $\operatorname{tmas}[1.690]=0.20$ & $\operatorname{tmas}[1.690]=0.20$ & $\operatorname{tmas}[1.690]=0.19$ & $\operatorname{tmas}[1.690]=0.19$ & $\operatorname{tmas}[1.690]=0.19$ \\
\hline $\operatorname{tmas}[2.690]=0.39$ & $\operatorname{tmas}[2.690]=0.38$ & $\operatorname{tmas}[2.690]=0.38$ & $\operatorname{tmas}[2.690]=0.38$ & $\operatorname{tmas}[2.690]=0.37$ \\
\hline $\operatorname{tmas}[3.690]=0.56$ & $\operatorname{tmas}[3.690]=0.55$ & $\operatorname{tmas}[3.690]=0.54$ & $\operatorname{tmas}[3.690]=0.53$ & $\operatorname{tmas}[3.690]=0.51$ \\
\hline $\operatorname{tmas}[4.690]=0.70$ & $\operatorname{tmas}[4.690]=0.68$ & $\operatorname{tmas}[4.690]=0.66$ & $\operatorname{tmas}[4.690]=0.64$ & $\operatorname{tmas}[4.690]=0.60$ \\
\hline $\operatorname{tmas}[5.690]=0.80$ & $\operatorname{tmas}[5.690]=0.77$ & $\operatorname{tmas}[5.690]=0.74$ & $\operatorname{tmas}[5.690]=0.69$ & $\operatorname{tmas}[5.690]=0.63$ \\
\hline $\operatorname{tmas}[6.690]=0.87$ & $\operatorname{tmas}[6.690]=0.82$ & $\operatorname{tmas}[6.690]=0.76$ & $\operatorname{tmas}[6.690]=0.69$ & $\operatorname{tmas}[6.690]=0.60$ \\
\hline $\operatorname{tmas}[7.690]=0.89$ & $\operatorname{tmas}[7.690]=0.82$ & $\operatorname{tmas}[7.690]=0.74$ & $\operatorname{tmas}[7.690]=0.64$ & $\operatorname{tmas}[7.690]=0.51$ \\
\hline $\operatorname{tmas}[8.690]=0.87$ & $\operatorname{tmas}[8.690]=0.77$ & $\operatorname{tmas}[8.690]=0.66$ & $\operatorname{tmas}[8.690]=0.53$ & $\operatorname{tmas}[8.690]=0.37$ \\
\hline $\operatorname{tmas}[9.690]=0.80$ & $\operatorname{tmas}[9.690]=0.68$ & $\operatorname{tmas}[9.690]=0.54$ & $\operatorname{tmas}[9.690]=0.38$ & $\operatorname{tmas}[9.690]=0.19$ \\
\hline $\operatorname{tmas}[10.690]=0.70$ & $\operatorname{tmas}[10.690]=0.55$ & $\operatorname{tmas}[10.690]=0.38$ & $\operatorname{tmas}[10.690]=0.19$ & $\operatorname{tmas}[10.690]=0.50$ \\
\hline $\operatorname{tmas}[11.690]=0.56$ & $\operatorname{tmas}[11.690]=0.38$ & $\operatorname{tmas}[11.690]=0.19$ & $\operatorname{tmas}[11.690]=0.85$ & \\
\hline $\operatorname{tmas}[12.690]=0.39$ & $\operatorname{tmas}[12.690]=0.20$ & $\operatorname{tmas}[12.690]=0.21$ & & \\
\hline
\end{tabular}


$\operatorname{tmas}[13.690]=0.20 \quad \operatorname{tmas}[13.690]=0.56$

$\operatorname{tmas}[14.690]=0.92$

The length of the cylinder is $l=10$.

Having analyzed the data of the table it can be concluded that there is a fact of an unjustified use of maximum possible number of the heating elements. Let us say if it is necessary to stabilize the temperature within 0.6 degrees it installation of 10 heating elements is sufficient, while a larger number will be redundant.

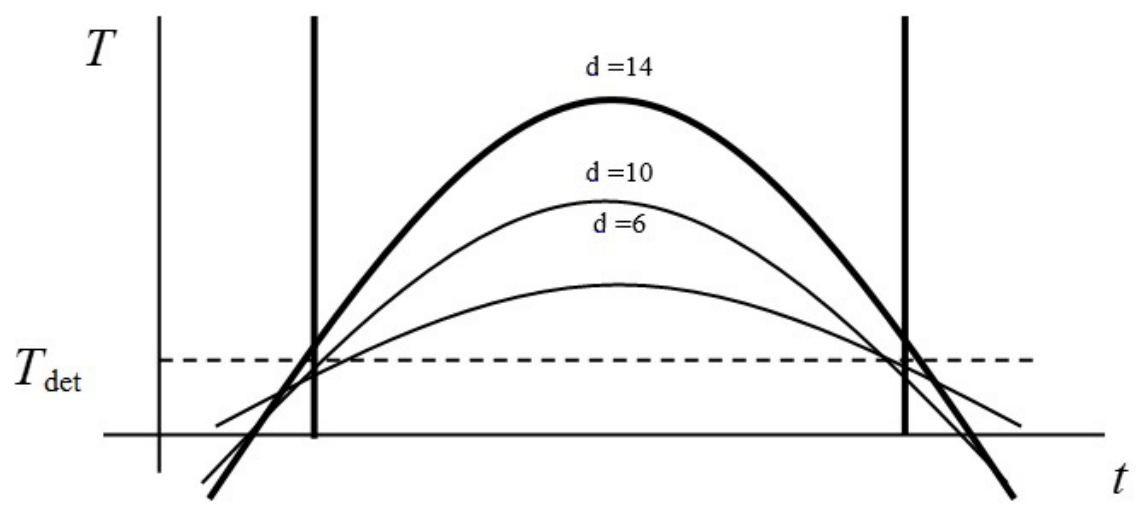

Figure 1. The value of the temperature field depending on time

Now it is necessary to understand whether the designed control system will behave the same in other initial parameters of the system. Particularly we are interested in the question of presence of hexagonal silicon-carbide as the control object. However, it is necessary to make simulation with different numbers of objects and different parameters. It will provide reliability of the obtained simulation results of the process of the temperature field stabilization. Let us conduct the research of the object, within the changing of its length. To do this, we will change the corresponding initial data: $l=0.5, k=10, d=9, x_{1}=\xi_{1}=1, T_{\text {det }}=0.3, \xi \in\{1,2,3,4,5,6,7,8,9\}$, $a^{2}=0.01$.

Table 2. The table calculation results by the number of sensors

\begin{tabular}{ccccc}
\hline$d=9$ & $d=8$ & $d=7$ & $d=6$ & $d=5$ \\
\hline $\operatorname{tmas}[1.690]=2.03$ & $\operatorname{tmas}[1.690]=2.01$ & $\operatorname{tmas}[1.690]=1.99$ & $\operatorname{tmas}[1.690]=1.95$ & $\operatorname{tmas}[1.690]=1.89$ \\
$\operatorname{tmas}[2.690]=3.82$ & $\operatorname{tmas}[2.690]=3.73$ & $\operatorname{tmas}[2.690]=3.59$ & $\operatorname{tmas}[2.690]=3.39$ & $\operatorname{tmas}[2.690]=3.07$ \\
$\operatorname{tmas}[3.690]=5.15$ & $\operatorname{tmas}[3.690]=4.87$ & $\operatorname{tmas}[3.690]=4.48$ & $\operatorname{tmas}[3.690]=3.91$ & $\operatorname{tmas}[3.690]=3.07$ \\
$\operatorname{tmas}[4.690]=5.86$ & $\operatorname{tmas}[4.690]=5.27$ & $\operatorname{tmas}[4.690]=4.48$ & $\operatorname{tmas}[4.690]=3.39$ & $\operatorname{tmas}[4.690]=1.89$ \\
$\operatorname{tmas}[5.690]=5.86$ & $\operatorname{tmas}[5.690]=4.87$ & $\operatorname{tmas}[5.690]=3.59$ & $\operatorname{tmas}[5.690]=1.95$ & $\operatorname{tmas}[5.690]=1.75$ \\
$\operatorname{tmas}[6.690]=5.15$ & $\operatorname{tmas}[6.690]=3.73$ & $\operatorname{tmas}[6.690]=1.99$ & $\operatorname{tmas}[6.690]=2.12$ & \\
$\operatorname{tmas}[7.690]=3.82$ & $\operatorname{tmas}[7.690]=2.01$ & $\operatorname{tmas}[7.690]=2.49$ & & \\
$\operatorname{tmas}[8.690]=2.03$ & $\operatorname{tmas}[8.690]=2.86$ & & & \\
$\operatorname{tmas}[9.690]=3.22$ & & & & \\
$d=14$ & $d=13$ & $d=12$ & & \\
$\operatorname{tmas}[1.570]=2.07$ & $\operatorname{tmas}[1.570]=2.06$ & $\operatorname{tmas}[1.570]=2.06$ & $\operatorname{tmas}[1.570]=2.05$ & $\operatorname{tmas}[1.570]=2.04$ \\
$\operatorname{tmas}[2.570]=4.04$ & $\operatorname{tmas}[2.570]=4.01$ & $\operatorname{tmas}[2.570]=3.98$ & $\operatorname{tmas}[2.570]=3.94$ & $\operatorname{tmas}[2.570]=3.89$ \\
$\operatorname{tmas}[3.570]=5.80$ & $\operatorname{tmas}[3.570]=5.73$ & $\operatorname{tmas}[3.570]=5.63$ & $\operatorname{tmas}[3.570]=5.51$ & $\operatorname{tmas}[3.570]=5.36$ \\
$\operatorname{tmas}[4.570]=7.28$ & $\operatorname{tmas}[4.570]=7.11$ & $\operatorname{tmas}[4.570]=6.90$ & $\operatorname{tmas}[4.570]=6.64$ & $\operatorname{tmas}[4.570]=6.30$ \\
$\operatorname{tmas}[5.570]=8.39$ & $\operatorname{tmas}[5.570]=8.08$ & $\operatorname{tmas}[5.570]=7.70$ & $\operatorname{tmas}[5.570]=7.22$ & $\operatorname{tmas}[5.570]=6.62$ \\
$\operatorname{tmas}[6.570]=9.08$ & $\operatorname{tmas}[6.570]=8.58$ & $\operatorname{tmas}[6.570]=7.97$ & $\operatorname{tmas}[6.570]=7.22$ & $\operatorname{tmas}[6.570]=6.30$ \\
$\operatorname{tmas}[7.570]=9.31$ & $\operatorname{tmas}[7.570]=8.58$ & $\operatorname{tmas}[7.570]=7.70$ & $\operatorname{tmas}[7.570]=6.64$ & $\operatorname{tmas}[7.570]=5.36$ \\
$\operatorname{tmas}[8.570]=9.08$ & $\operatorname{tmas}[8.570]=8.08$ & $\operatorname{tmas}[8.570]=6.90$ & $\operatorname{tmas}[8.570]=5.51$ & $\operatorname{tmas}[8.570]=3.89$ \\
$\operatorname{tmas}[9.570]=8.39$ & $\operatorname{tmas}[9.570]=7.11$ & $\operatorname{tmas}[9.570]=5.63$ & $\operatorname{tmas}[9.570]=3.94$ & $\operatorname{tmas}[9.570]=2.04$ \\
$\operatorname{tmas}[10.570]=7.28$ & $\operatorname{tmas}[10.570]=5.73$ & $\operatorname{tmas}[10.570]=3.98$ & $\operatorname{tmas}[10.570]=2.05$ & $\operatorname{tmas}[10.570]=3.59$ \\
$\operatorname{tmas}[11.570]=5.80$ & $\operatorname{tmas}[11.570]=4.01$ & $\operatorname{tmas}[11.570]=2.06$ & $\operatorname{tmas}[11.570]=3.95$ & \\
\hline & & & &
\end{tabular}




\begin{tabular}{|c|c|c|}
\hline $\operatorname{tmas}[12.570]=4.04$ & $\operatorname{tmas}[12.570]=2.06$ & $\operatorname{tmas}[12.570]=4.32$ \\
\hline $\operatorname{tmas}[13.570]=2.07$ & $\operatorname{tmas}[13.570]=4.68$ & \\
\hline $\operatorname{tmas}[14.570]=2.07$ & & \\
\hline
\end{tabular}

It is clear that the achieving the predetermined temperature at the predetermined range depends on the material of the rod as well. Let us carry out a research for an identical object, but made of other material. Let us change the initial data to meet the new requirements and set the following values: $l=10, k=10, d=9, x_{1}=\xi_{1}=1, T_{\text {det }}=$ $0.3, \xi \in\{1,2,3,4,5,6,7,8,9\}, a^{2}=0.2$.

Let us carry out a research for materials with the lowest thermal conductivity, in order to compare results.

Table 3. The table calculation results by the number of sensors

\begin{tabular}{lllll}
\hline$d=9$ & $d=8$ & $d=7$ & $d=6$ & $d=5$ \\
\hline $\operatorname{tmas}[1.690]=0.11$ & $\operatorname{tmas}[1.690]=0.37$ & $\operatorname{tmas}[1.690]=0.33$ & $\operatorname{tmas}[1.690]=0.23$ & $\operatorname{tmas}[1.690]=0.24$ \\
$\operatorname{tmas}[2.690]=0.21$ & $\operatorname{tmas}[2.690]=0.57$ & $\operatorname{tmas}[2.690]=0.60$ & $\operatorname{tmas}[2.690]=0.38$ & $\operatorname{tmas}[2.690]=0.39$ \\
$\operatorname{tmas}[3.690]=0.29$ & $\operatorname{tmas}[3.690]=0.59$ & $\operatorname{tmas}[3.690]=0.73$ & $\operatorname{tmas}[3.690]=0.43$ & $\operatorname{tmas}[3.690]=0.39$ \\
$\operatorname{tmas}[4.690]=0.33$ & $\operatorname{tmas}[4.690]=0.58$ & $\operatorname{tmas}[4.690]=0.73$ & $\operatorname{tmas}[4.690]=0.38$ & $\operatorname{tmas}[4.690]=0.24$ \\
$\operatorname{tmas}[5.690]=0.33$ & $\operatorname{tmas}[5.690]=0.59$ & $\operatorname{tmas}[5.690]=0.60$ & $\operatorname{tmas}[5.690]=0.23$ & $\operatorname{tmas}[5.690]=0.24$ \\
$\operatorname{tmas}[6.690]=0.29$ & $\operatorname{tmas}[6.690]=0.57$ & $\operatorname{tmas}[6.690]=0.33$ & $\operatorname{tmas}[6.690]=0.84$ & \\
$\operatorname{tmas}[7.690]=0.21$ & $\operatorname{tmas}[7.690]=0.37$ & $\operatorname{tmas}[7.690]=0.41$ & & \\
$\operatorname{tmas}[8.690]=0.11$ & $\operatorname{tmas}[8.690]=0.02$ & & & \\
$\operatorname{tmas}[9.690]=0.84$ & & & & \\
$d=14$ & $d=13$ & $d=12$ & & \\
$\operatorname{tmas}[1.690]=0.19$ & $\operatorname{tmas}[1.690]=0.17$ & $\operatorname{tmas}[1.690]=0.24$ & $\operatorname{tmas}[1.690]=0.09$ & $\operatorname{tmas}[1.690]=0.10$ \\
$\operatorname{tmas}[2.690]=0.38$ & $\operatorname{tmas}[2.690]=0.33$ & $\operatorname{tmas}[2.690]=0.45$ & $\operatorname{tmas}[2.690]=0.19$ & $\operatorname{tmas}[2.690]=0.19$ \\
$\operatorname{tmas}[3.690]=0.52$ & $\operatorname{tmas}[3.690]=0.46$ & $\operatorname{tmas}[3.690]=0.62$ & $\operatorname{tmas}[3.690]=0.26$ & $\operatorname{tmas}[3.690]=0.26$ \\
$\operatorname{tmas}[4.690]=0.64$ & $\operatorname{tmas}[4.690]=0.55$ & $\operatorname{tmas}[4.690]=0.74$ & $\operatorname{tmas}[4.690]=0.32$ & $\operatorname{tmas}[4.690]=0.31$ \\
$\operatorname{tmas}[5.690]=0.71$ & $\operatorname{tmas}[5.690]=0.61$ & $\operatorname{tmas}[5.690]=0.80$ & $\operatorname{tmas}[5.690]=0.34$ & $\operatorname{tmas}[5.690]=0.33$ \\
$\operatorname{tmas}[6.690]=0.75$ & $\operatorname{tmas}[6.690]=0.63$ & $\operatorname{tmas}[6.690]=0.82$ & $\operatorname{tmas}[6.690]=0.34$ & $\operatorname{tmas}[6.690]=0.31$ \\
$\operatorname{tmas}[7.690]=0.76$ & $\operatorname{tmas}[7.690]=0.63$ & $\operatorname{tmas}[7.690]=0.80$ & $\operatorname{tmas}[7.690]=0.32$ & $\operatorname{tmas}[7.690]=0.26$ \\
$\operatorname{tmas}[8.690]=0.75$ & $\operatorname{tmas}[8.690]=0.61$ & $\operatorname{tmas}[8.690]=0.74$ & $\operatorname{tmas}[8.690]=0.26$ & $\operatorname{tmas}[8.690]=0.19$ \\
$\operatorname{tmas}[9.690]=0.71$ & $\operatorname{tmas}[9.690]=0.55$ & $\operatorname{tmas}[9.690]=0.62$ & $\operatorname{tmas}[9.690]=0.19$ & $\operatorname{tmas}[9.690]=0.10$ \\
$\operatorname{tmas}[10.690]=0.64$ & $\operatorname{tmas}[10.690]=0.46$ & $\operatorname{tmas}[10.690]=0.45$ & $\operatorname{tmas}[10.690]=0.09$ & $\operatorname{tmas}[10.690]=0.82$ \\
$\operatorname{tmas}[11.690]=0.52$ & $\operatorname{tmas}[11.690]=0.33$ & $\operatorname{tmas}[11.690]=0.24$ & $\operatorname{tmas}[11.690]=0.93$ & \\
$\operatorname{tmas}[12.690]=0.38$ & $\operatorname{tmas}[12.690]=0.17$ & $\operatorname{tmas}[12.690]=0.69$ & & \\
$\operatorname{tmas}[13.690]=0.19$ & $\operatorname{tmas}[13.690]=-0.70$ & & & \\
$\operatorname{tmas}[14.690]=0.61$ & & & &
\end{tabular}

The material thermal conductivity $a^{2}=0.2$.

The length of the cylinder is $l=10$.

Table 4. The table calculation results by the number of sensors

\begin{tabular}{llllc}
\hline$d=9$ & $d=8$ & $d=7$ & $d=6$ & $d=5$ \\
\hline $\operatorname{tmas}[1.690]=0.89$ & $\operatorname{tmas}[1.690]=0.81$ & $\operatorname{tmas}[1.690]=0.77$ & $\operatorname{tmas}[1.690]=0.82$ & $\operatorname{tmas}[1.690]=0.99$ \\
$\operatorname{tmas}[2.690]=0.90$ & $\operatorname{tmas}[2.690]=0.77$ & $\operatorname{tmas}[1.690]=0.60$ & $\operatorname{tmas}[1.690]=0.55$ & $\operatorname{tmas}[1.690]=0.99$ \\
$\operatorname{tmas}[3.690]=0.89$ & $\operatorname{tmas}[3.690]=0.83$ & $\operatorname{tmas}[1.690]=0.74$ & $\operatorname{tmas}[1.690]=0.45$ & $\operatorname{tmas}[1.690]=0.99$ \\
$\operatorname{tmas}[4.690]=0.90$ & $\operatorname{tmas}[4.690]=0.76$ & $\operatorname{tmas}[1.690]=0.74$ & $\operatorname{tmas}[1.690]=0.55$ & $\operatorname{tmas}[1.690]=0.99$ \\
$\operatorname{tmas}[5.690]=0.90$ & $\operatorname{tmas}[5.690]=0.83$ & $\operatorname{tmas}[1.690]=0.60$ & $\operatorname{tmas}[1.690]=0.82$ & $\operatorname{tmas}[5.690]=0.64$ \\
$\operatorname{tmas}[6.690]=0.89$ & $\operatorname{tmas}[6.690]=0.77$ & $\operatorname{tmas}[1.690]=0.77$ & $\operatorname{tmas}[1.690]=0.40$ & \\
$\operatorname{tmas}[7.690]=0.90$ & $\operatorname{tmas}[7.690]=0.81$ & $\operatorname{tmas}[1.690]=0.28$ & & \\
$\operatorname{tmas}[8.690]=0.89$ & $\operatorname{tmas}[8.690]=0.02$ & & & \\
$\operatorname{tmas}[9.690]=0.82$ & & & & \\
$d=14$ & $d=13$ & $d=12$ & $d=11$ & $d=10$ \\
$\operatorname{tmas}[1.690]=1.35$ & $\operatorname{tmas}[1.690]=1.27$ & $\operatorname{tmas}[1.890]=1.19$ & $\operatorname{tmas}[1.690]=1.09$ & $\operatorname{tmas}[1.690]=0.99$ \\
\hline
\end{tabular}




$\begin{array}{lllll}\operatorname{tmas}[2.690]=1.47 & \operatorname{tmas}[2.690]=1.34 & \operatorname{tmas}[2.890]=1.21 & \operatorname{tmas}[2.690]=1.10 & \operatorname{tmas}[2.690]=1.00 \\ \operatorname{tmas}[3.690]=1.32 & \operatorname{tmas}[3.690]=1.25 & \operatorname{tmas}[3.890]=1.18 & \operatorname{tmas}[3.690]=1.09 & \operatorname{tmas}[3.690]=0.99 \\ \operatorname{tmas}[4.690]=1.44 & \operatorname{tmas}[4.690]=1.34 & \operatorname{tmas}[4.890]=1.22 & \operatorname{tmas}[4.690]=1.10 & \operatorname{tmas}[4.690]=1.00 \\ \operatorname{tmas}[5.690]=1.39 & \operatorname{tmas}[5.690]=1.26 & \operatorname{tmas}[5.890]=1.17 & \operatorname{tmas}[5.690]=1.09 & \operatorname{tmas}[5.690]=0.99 \\ \operatorname{tmas}[6.690]=1.36 & \operatorname{tmas}[6.690]=1.31 & \operatorname{tmas}[6.890]=1.22 & \operatorname{tmas}[6.690]=1.09 & \operatorname{tmas}[6.690]=1.00 \\ \operatorname{tmas}[7.690]=1.44 & \operatorname{tmas}[7.690]=1.31 & \operatorname{tmas}[7.890]=1.17 & \operatorname{tmas}[7.690]=1.10 & \operatorname{tmas}[7.690]=0.99 \\ \operatorname{tmas}[8.690]=1.36 & \operatorname{tmas}[8.690]=1.26 & \operatorname{tmas}[8.890]=1.22 & \operatorname{tmas}[8.690]=1.09 & \operatorname{tmas}[8.690]=1.00 \\ \operatorname{tmas}[9.690]=1.39 & \operatorname{tmas}[9.690]=1.34 & \operatorname{tmas}[9.890]=1.18 & \operatorname{tmas}[9.690]=1.10 & \operatorname{tmas}[9.690]=0.99 \\ \operatorname{tmas}[10.690]=1.44 & \operatorname{tmas}[10.690]=1.25 & \operatorname{tmas}[10.890]=1.21 & \operatorname{tmas}[10.690]=1.09 & \operatorname{tmas}[10.690]=0.91 \\ \operatorname{tmas}[11.690]=1.32 & \operatorname{tmas}[11.690]=1.34 & \operatorname{tmas}[11.890]=1.19 & \operatorname{tmas}[11.690]=0.87 & \\ \operatorname{tmas}[12.690]=1.47 & \operatorname{tmas}[12.690]=1.27 & \operatorname{tmas}[12.890]=0.76 & & \\ \operatorname{tmas}[13.690]=1.35 & \operatorname{tmas}[13.690]=0.58 & & & \\ \operatorname{tmas}[14.690]=0.37 & & & & \end{array}$

The material thermal conductivity $a^{2}=0.00001$.

The length of the cylinder is $l=10$.

The results show that maintaining the predetermined temperature in the rod depends on the material type and on the number of discretely located heating elements. It can be also concluded that an excessively large number of impulse heating elements is capable to exceed the predetermined temperature range. This is due to the fact that the heating impulse comes before the material has time to cool, thus causing overheating. Too small amount makes it impossible to heat the entire rod up to the required temperature.

$$
\begin{gathered}
\frac{\partial T}{\partial t}=a^{2}\left(\frac{\partial^{2} T}{\partial x^{2}}+\frac{\partial^{2} T}{\partial y^{2}}+\frac{\partial^{2} T}{\partial z^{2}}\right) ; \\
0<x<l_{x} ; \quad 0<y<l_{y} ; 0<z<l_{z} .
\end{gathered}
$$

Boundary and initial conditions of the control object:

$$
\begin{gathered}
T(x, y, \tau)=U(x, y, \tau) ; \frac{\partial T(x, y, 0, \tau)}{\partial r}=0 ; \\
T(x, 0, \tau)=T\left(x, l_{y}, \tau\right)=T(0, y, \tau)=T\left(l_{x}, y, \tau\right)=0 \\
T(x, y, 0)=0 ;
\end{gathered}
$$

The values of the temperature field are determined using the Green's function, which in two-dimensional form takes the form of:

$$
\begin{aligned}
& G(x, y, \rho, v, t)=\frac{4}{l_{1} \cdot l_{2}} \cdot \sum_{k, m=1}^{\infty} \sin \left(\frac{k \cdot \pi \cdot x}{l_{1}}\right) \cdot \sin \left(\frac{m \cdot \pi \cdot y}{l_{2}}\right) \cdot \sin \left(\frac{k \cdot \pi \cdot \rho}{l_{1}}\right) \times \\
& \times \sin \left(\frac{m \cdot \pi \cdot v}{l_{2}}\right) \cdot \exp \left[-a^{2} \pi^{2} \cdot t \cdot\left(\frac{k^{2}}{l_{1}^{2}}+\frac{m^{2}}{l_{2}^{2}}\right)\right]
\end{aligned}
$$

In this function, the coordinates of the heating points are $x, y$, geometrical indexes of the control object are $1_{1}, 1_{2}$; the number of the members in the Fourier's series $k, m$; temperature sensor coordinates are $\mathrm{p}, v$. 


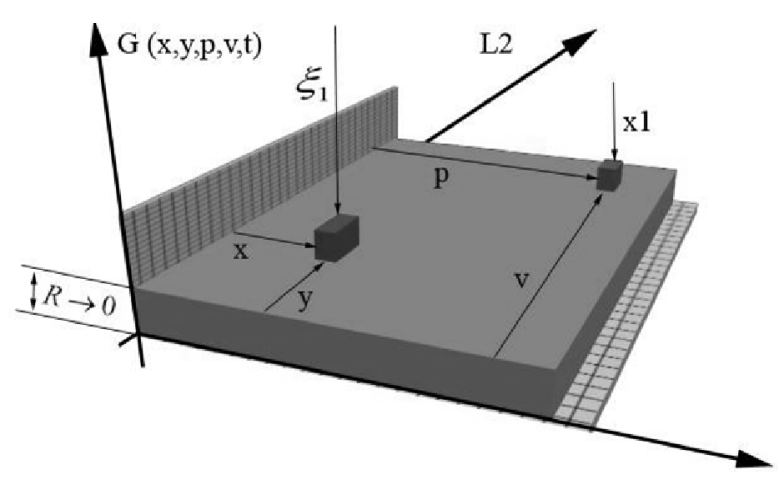

L1

Figure 2. The regarded object

Let's consider the system at the initial time point $n \rightarrow \infty, t=0, \tau=0$. The effect of the Fourier's series constituents will have the form of:

$$
\exp \left[-a^{2} \pi^{2} \cdot t \cdot\left(\frac{k^{2}}{l_{1}^{2}}+\frac{m^{2}}{l_{2}^{2}}\right)\right]=1 ;
$$

The value of the temperature field in the spatially distributed control object will have the form of:

$$
T(x, y, \xi)=\frac{4}{l_{1} \cdot l_{2}} \cdot \sum_{k, m=1}^{\infty} \sin \left(\frac{k \cdot \pi}{l_{1}}\right) x \cdot \sin \left(\frac{k \cdot \pi}{l_{1}}\right) p \cdot \sin \left(\frac{m \cdot \pi}{l_{2}}\right) y \cdot \sin \left(\frac{m \cdot \pi}{l_{2}}\right) v ;
$$

The impulse source creates the heating impulse in a point $p=v=\frac{l}{4}$, then the value of the temperature field will have the form of:

$$
T(x, y, \xi)=\frac{4}{l_{1} \cdot l_{2}} \cdot \sum_{k, m=1}^{\infty} \sin \left(\frac{k \cdot \pi}{l_{1}}\right) x \cdot \sin \left(\frac{k \cdot \pi}{4}\right) \cdot \sin \left(\frac{m \cdot \pi}{l_{2}}\right) y \cdot \sin \left(\frac{m \cdot \pi}{4}\right)
$$

For this function, the amplitude of the Fourier's series takes the form of: $A_{n}=\frac{4}{l_{1} \cdot l_{2}}\left|\sin \frac{\pi p}{4} \cdot \sin \frac{\pi v}{4}\right|$. Let us conduct the calculation of the function, in which we take into account only the first 5 members. Within this calculation we will set the following conditions (Zarembo, et al. (2004); Rapoport, \& Pleshivtseva (2010); Pleshivtseva, \& Rapoport (2009); Rapoport (2006); Rapoport, \& Pleshivtseva (2006); Rapoport (1996); 1Ilyushin, et al. (2014)).

$$
x=y=\frac{l}{4}, p=v=1,2,3,4,5
$$

Then:

$$
\begin{gathered}
T_{1}(x, y)=\frac{4}{l_{1} \cdot l_{2}}\left(\sin \frac{\pi}{4} \sin \frac{\pi}{l_{1}} x\right) \cdot\left(\sin \frac{\pi}{4} \sin \frac{\pi}{l_{2}} y\right) ; A_{1}=32 \frac{1}{l^{2}} ; \\
T_{2}(x, y)=\frac{4}{l_{1} \cdot l_{2}}\left(\sin \frac{2 \pi}{4} \sin \frac{2 \pi}{l_{1}} x\right) \cdot\left(\sin \frac{2 \pi}{4} \sin \frac{2 \pi}{l_{2}} y\right) ; A_{2}=64 \frac{1}{l^{2}} \\
T_{3}(x, y)=\frac{4}{l_{1} \cdot l_{2}}\left(\sin \frac{3 \pi}{4} \sin \frac{3 \pi}{l_{1}} x\right) \cdot\left(\sin \frac{3 \pi}{4} \sin \frac{3 \pi}{l_{2}} y\right) ; A_{3}=32 \frac{1}{l^{2}} ; \\
T_{4}(x, y)=\frac{4}{l_{1} \cdot l_{2}}\left(\sin \frac{4 \pi}{4} \sin \frac{4 \pi}{l_{1}} x\right) \cdot\left(\sin \frac{4 \pi}{4} \sin \frac{4 \pi}{l_{2}} y\right) ; A_{4}=\frac{1}{l^{2}}
\end{gathered}
$$




$$
T_{5}(x, y)=\frac{4}{l_{1} \cdot l_{2}}\left(\sin \frac{5 \pi}{4} \sin \frac{5 \pi}{l_{1}} x\right) \cdot\left(\sin \frac{5 \pi}{4} \sin \frac{5 \pi}{l_{2}} y\right) ; A_{5}=32 \frac{1}{l^{2}} ;
$$

From the condition we can see that $G(x, y, \rho, v, t) \geq 0$, at any $x, y, \mathrm{p}, \mathrm{v}, t$. Then it is seen that the range of the positive values of the function in the range of $x$, will decrease. The restriction will cause the system to the solutions region near the point $p=v=\frac{l}{4}$, at $p, v \rightarrow \infty$ we will receive

$$
\frac{4}{l_{1} \cdot l_{2}} \cdot \sum_{k, m=1}^{\infty} \sin \left(\frac{k \cdot \pi}{l_{1}}\right) x \cdot \sin \left(\frac{k \cdot \pi}{l_{1}}\right) p \cdot \sin \left(\frac{m \cdot \pi}{l_{2}}\right) y \cdot \sin \left(\frac{m \cdot \pi}{l_{2}}\right) v
$$

spatially distributed delta function in the form of Fourier's series, i.e.

$$
\delta(x-p, y-v)=\frac{4}{l_{1} \cdot l_{2}} \cdot \sum_{k, m=1}^{\infty} \sin \left(\frac{k \cdot \pi}{l_{1}}\right) x \cdot \sin \left(\frac{k \cdot \pi}{l_{1}}\right) p \cdot \sin \left(\frac{m \cdot \pi}{l_{2}}\right) y \cdot \sin \left(\frac{m \cdot \pi}{l_{2}}\right) v .
$$

Such $\delta$ - function, which is presented as the Fourier's series is considered as the generalized one. These functions are of great use in systems with distributed parameters, in particular for their simulation.

The arguments of these functions are usually coordinates of the control object.

$$
\delta(\mathrm{x}-\mathrm{p}, \mathrm{y}-\mathrm{v})=\left\{\begin{array}{ccc}
\infty, & \text { as } & y=v \\
0, & \text { as } & y \neq v \\
\infty, & \text { as } & x=p \\
0, & \text { as } & x \neq p
\end{array}\right.
$$

Within the regarding of the $f(x, y)$ function, which has the spatial distribution $\left[0, l_{1}\right]\left[0, l_{2}\right]$, the following equation is right:

$$
\int_{0}^{l_{1}} \int_{0}^{l_{2}} f(x, y) \delta(x-p, y-v) d x \cdot d y=f(p, v)
$$

Let us describe the impulses limited by consumption by the input of the time $\delta-$ function $\delta(t-\tau)$. In this case, we find that the infinite impact at the point of distributed control object will be an ideal $\delta$-function. Then $\delta(t-\tau)=0$ at any $t$. However, in the case of $t=\tau$, the function will turn to the perpetuity then

$$
\int \delta(t-\tau) d t=1
$$

If the following function takes place, $f(t)$, which ${ }^{-\infty}$ determined in the section $\left[t_{0}, t\right]$, then

$$
\int_{t_{0}}^{t} f(t) \delta(t-\tau) d t=\left\{\begin{array}{cc}
f(\tau), \text { if } & \tau \in\left[t_{0}, t\right] \\
0, \text { if } & \tau \notin\left[t_{0}, t\right]
\end{array}\right.
$$

We will have that for the description of the function and $\delta$ - function, the presence of three variables, namely, the coordinate and time variables. On that basis, we have, that three-dimension $\delta$ - function $\delta(x-p) \delta(y-v) \delta(t-\tau)$ will change their integral representation, and namely they will take the form below:

$$
\int_{0}^{L_{1}} \int_{0}^{L} \int_{t_{0}}^{t} f(x, y, t) \cdot \delta(y-v) \cdot \delta(x-p) \cdot \delta(t-\tau) \cdot d x \cdot d y \cdot d t=f(p, v, \tau)
$$

In the case, if to the distributed control object the effect is applied with the relay control principle there the change of the output function will occur. Let us apply the effect at the point $p_{0}, v_{0}$ at the moment of time $\tau_{0}$, and then we will receive the following function:

$$
T(x, y, t)=\int_{0}^{t} \int_{0}^{L} \int_{0}^{L} G(x, y, t, p, v, \tau) \delta\left(p-p_{0}\right) \delta\left(v-v_{0}\right) \times
$$




$$
\times \delta\left(\tau-\tau_{0}\right) d p d v d \tau=G\left(x, y, t, p_{0}, v, \tau_{0}\right)
$$

For the further analysis, we will fix the time at nonzero point. And, as it was in the previous example, we will analyze the amplitude of the Fourier's series, as follows:

$$
A_{n}=\left|\frac{4}{l_{1} \cdot l_{2}} \sin \frac{\pi n}{l_{1}} p \cdot \sin \frac{\pi n}{l_{2}} v \cdot \exp \left[-a^{2} \pi^{2} \cdot t \cdot\left(\frac{k^{2}}{l_{1}^{2}}+\frac{m^{2}}{l_{2}^{2}}\right)\right]\right|
$$

In the case of $n \rightarrow \infty$, then the effect of each following member of the Fourier's series is reduced. And, therefore, the number of members of the Fourier's series can be limited. This restriction does not affect the model of the system, as each following member has less influence, but to exclude the possibility of changing of the initial model we will analyze the error calculations. This calculation will be carried out on the basis of a specified accuracy $\varepsilon$.

If we conduct the accuracy calculation, as $l=10, \mathrm{a}=0.01, t=10000, \varepsilon=0.0001$; then $n \geq 9.167$, and, consequently, in the case of system requirements has the necessary accuracy of calculations $\varepsilon=0.0001$, the accuracy of the result is $91 \%$ within the calculation of the nine members of the Fourier series. You can also see that in the course of time, the system goes to the stable operation regime and the amplitude decreases. Then we find that in the steady-state members of the Fourier's series will have the least impact. Thereby, let us calculate the time point, after which the number of the Fourier series can be reduced. For the calculation we take the same values as in the previous example:

$$
t \geq\left(\frac{l}{\pi n a}\right)^{2} \ln \frac{4}{\varepsilon l}
$$

The considered model describes the behavior of the temperature field in the static mode without interaction between sources and sensors of the system. Let us analyze the system in a dynamic mode. For that we will receive the control, variable in space function of the initial heating. This function allows determining the values of the temperature field after the accounting of the effects of all heat sources on the object. Also, this function will allow to simulating the behavior of the temperature field in the course of time. Let us consider the plane, carrying the $n$ number of point impulse sources and $n$ number of sensors and set the following boundary conditions

$$
T(0, y, t)=T\left(l_{1}, y, t\right)=0 ; T(x, 0, t)=T\left(x, l_{2}, t\right)=0 ; T(x, y, 0)=0 .
$$

The system goes out from the state of rest under the action of impulse heating elements with relay control principle. All heating elements are activated simultaneously $\tau_{0}=0$. All of these heating elements will impact on all the temperature sensors of the system. If we consider the case of the influence of all sources into one sensor, the cumulative effect would be as follows:

$$
\begin{aligned}
& T\left(x_{1}, y, t, \tau_{0}\right)=\sum_{i=1}^{n} \frac{4}{l_{1} \cdot l_{2}} \sum_{k, m=1}^{\infty}\left[-a^{2} \pi^{2} \cdot t \cdot\left(\frac{k^{2}}{l_{1}^{2}}+\frac{m^{2}}{l_{2}^{2}}\right)\right] \cdot \sin \left(\frac{k \cdot \pi \cdot x}{l_{1}}\right) \cdot \sin \left(\frac{m \cdot \pi \cdot y}{l_{2}}\right) \times \\
& \times \sin \left(\frac{k \cdot \pi \cdot \rho}{l_{1}}\right) \cdot \sin \left(\frac{m \cdot \pi \cdot v}{l_{2}}\right) ;
\end{aligned}
$$

In this formula, the time variable heating elements $\xi_{1}, \xi_{2}$ create heating impulses in temperature sensors x1, x2. As the system is not in well-stable regime, created heating impulse has maximum amplitude. The temperature field in the object extends annularly. The next standing heating elements create the tension point at the contact points of the temperature fields. For the analysis of the annular distribution and interaction between the impulses let us calculate the behavior of the temperature field near the hot spot $\mathrm{x}(1 ; 1)$, and the coordinate of the temperature sensor $\mathrm{p}(1 ; 1)\left(\right.$ Chernishev, 2009). With the following input data: $l=10, a=0.00001, x_{1}=\xi_{1}=1$, $T_{\text {det }}=0,3, k=10, d=9 . \xi_{i} \in\{1,2,3,4,5,6,7,8,9\}$,

Then: 


$$
\begin{aligned}
& T\left(x_{1}, y_{2}, t, \tau_{0}\right)=\sum_{i=1}^{n} \frac{4}{l_{1} \cdot l_{2}} \sum_{k, m=1}^{\infty}\left[-a^{2} \pi^{2} \cdot t \cdot\left(\frac{k^{2}}{l_{1}^{2}}+\frac{m^{2}}{l_{2}^{2}}\right)\right] \cdot \sin \left(\frac{k \cdot \pi \cdot x}{l_{1}}\right) \cdot \sin \left(\frac{m \cdot \pi \cdot y}{l_{2}}\right) \times \\
& \times \sin \left(\frac{k \cdot \pi \cdot \rho}{l_{1}}\right) \cdot \sin \left(\frac{m \cdot \pi \cdot v}{l_{2}}\right)=4.8827000 ; \\
& T\left(x_{1}, y_{0}, t, \tau_{0}\right)=\sum_{i=1}^{n} \frac{4}{l_{1} \cdot l_{2}} \sum_{k, m=1}^{\infty}\left[-a^{2} \pi^{2} \cdot t \cdot\left(\frac{k^{2}}{l_{1}^{2}}+\frac{m^{2}}{l_{2}^{2}}\right)\right] \cdot \sin \left(\frac{k \cdot \pi \cdot x}{l_{1}}\right) \cdot \sin \left(\frac{m \cdot \pi \cdot y}{l_{2}}\right) \times \\
& \times \sin \left(\frac{m \cdot \pi \cdot v}{l_{2}}\right) \cdot \sin \left(\frac{k \cdot \pi \cdot \rho}{l_{1}}\right)=4.8827316 ; \\
& T\left(x_{2}, y_{1}, t, \tau_{0}\right)=\sum_{i=1}^{n} \frac{4}{l_{1} \cdot l_{2}} \sum_{k, m=1}^{\infty}\left[-a^{2} \pi^{2} \cdot t \cdot\left(\frac{k^{2}}{l_{1}^{2}}+\frac{m^{2}}{l_{2}^{2}}\right)\right] \cdot \sin \left(\frac{k \cdot \pi \cdot x}{l_{1}}\right) \cdot \sin \left(\frac{m \cdot \pi \cdot y}{l_{2}}\right) \times \\
& \times \sin \left(\frac{m \cdot \pi \cdot v}{l_{2}}\right) \cdot \sin \left(\frac{k \cdot \pi \cdot \rho}{l_{1}}\right)=4.8827116 ; \\
& T\left(x_{0}, y_{1}, t, \tau_{0}\right)=\sum_{i=1}^{n} \frac{4}{l_{1} \cdot l_{2}} \sum_{k, m=1}^{\infty}\left[-a^{2} \pi^{2} \cdot t \cdot\left(\frac{k^{2}}{l_{1}^{2}}+\frac{m^{2}}{l_{2}^{2}}\right)\right] \cdot \sin \left(\frac{k \cdot \pi \cdot x}{l_{1}}\right) \cdot \sin \left(\frac{m \cdot \pi \cdot y}{l_{2}}\right) \times \\
& \times \sin \left(\frac{m \cdot \pi \cdot v}{l_{2}}\right) \cdot \sin \left(\frac{k \cdot \pi \cdot \rho}{l_{1}}\right)=4.8827222 ;
\end{aligned}
$$

Let us display the graphically obtained results (see. Figure 3) The chart shows the total amplitude of the temperature field. However, in the course of time a drop of temperature is occurred and there is a need to find a function of the dynamic display of the temperature field behavior:

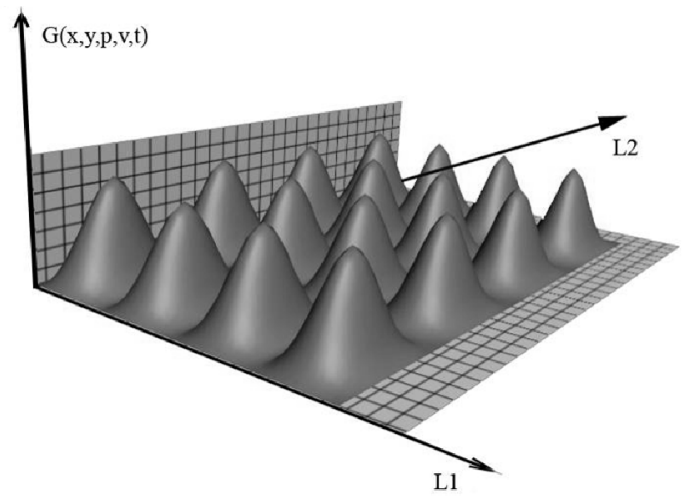

Figure 3. The first heating impulse

For the dynamic display of the behavior of the thermal field we will apply the following function:

$$
\begin{aligned}
& T\left(x_{j}, y_{j}, t\right)=\sum_{i=1}^{d} \sum_{k, m=1}^{\infty} \frac{4}{l_{1} \cdot l_{2}} \exp \left[-a^{2} \pi^{2} \cdot t \cdot\left(\frac{k^{2}}{l_{1}^{2}}+\frac{m^{2}}{l_{2}^{2}}\right)\right] \cdot \sin \left(\frac{k \cdot \pi \cdot x_{j}}{l_{1}}\right) \times \\
& \times \sin \left(\frac{k \cdot \pi \cdot \rho_{i}}{l_{1}}\right) \cdot \cdot \sin \left(\frac{m \cdot \pi \cdot y_{j}}{l_{2}}\right) \cdot \sin \left(\frac{m \cdot \pi \cdot v_{i}}{l_{2}}\right)+\sum_{p} \sum_{k, m=1}^{\infty} \frac{4}{l_{1} \cdot l_{2}} \times \\
& \times \exp \left[-a^{2} \pi^{2} \cdot\left(t-\tau_{p}\right) \cdot\left(\frac{k^{2}}{l_{1}^{2}}+\frac{m^{2}}{l_{2}^{2}}\right)\right] \sin \left(\frac{m \cdot \pi \cdot y_{j}}{l_{2}}\right) \cdot \sin \left(\frac{k \cdot \pi \cdot x_{j}}{l_{1}}\right) \times \\
& \times \sin \left(\frac{k \cdot \pi \cdot \rho_{z(p)}}{l_{1}}\right) \cdot \sin \left(\frac{m \cdot \pi \cdot v_{z(p)}}{l_{2}}\right) ;
\end{aligned}
$$

where the number of impulse elements with relay control principle is denoted by variable $-d$, and its serial number $-p=1,2,3 \ldots$; through the variable $z(p)$ let us show one of the impulse elements with relay control principle; $\tau_{\mathrm{p}}$ - the time point of the power source switching on is under number $z(p) ; 1_{1}, 1_{2}-$ spatial coordinates of the distributed control object; 
The obtained function of the initial heating is able to determine the behavior of the temperature field with the course of time at any point of the control object (Chernyshev, 2009).

Let us consider the interaction of thermal fields in a two-dimensional object and the type of material, wich is characterized by the $\mathrm{a}^{2}$ thermal conductivity. Let us assume that the rod is negligibly thin, that is, we will take it as one-dimensional. Let us solve the problem of synthesis of control system, the stabilization of the temperature field. To do this, let us implement the Green's function in the Delphi language and write a program that simulates the behavior of the temperature field in the course of time. The program code that implements this task is as follows:

Begin

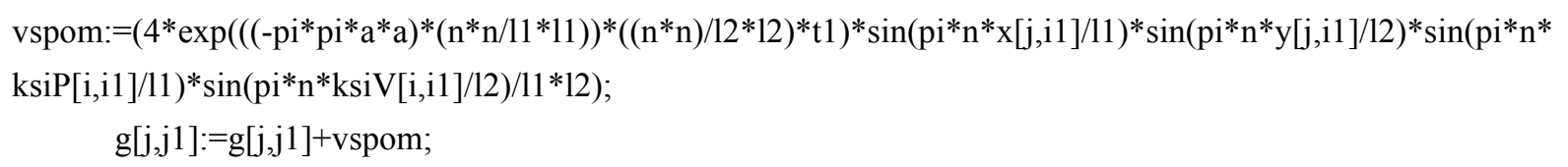

End;

End;

$\mathrm{t} \_\operatorname{mas}[\mathrm{j}, \mathrm{t}]:=\mathrm{g}[\mathrm{j}]+\mathrm{q}[\mathrm{j}]$;

listbox 1.Items.Add('tmas['+IntToStr(j)+','+IntToStr(t)+']='+floatToStr(t_mas[j,t]));

$\mathrm{c}:=\mathrm{c}+1$;

end;

end;

for $\mathrm{j}:=1$ to $\mathrm{d}$ do

begin

for $\mathrm{t}:=1$ to $2001 \mathrm{do}$

begin

listbox2.Items.Add('t['+IntToStr(j)+','+IntToStr(t-1)+']='+floatToStr(t_mas[j,t]));

end;

end;

In the course of time the function has a form of:

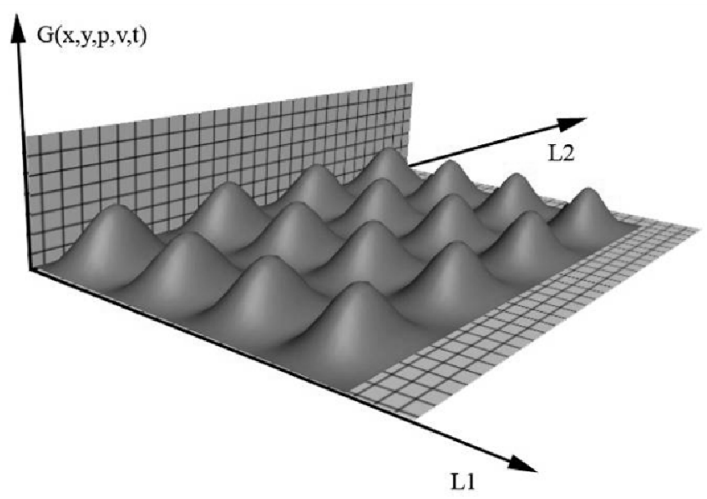

Figure 4. The system in the process of the stationary state engagement

After which the system will pass to the stationary state of the system. Namely, stable temperature regimes will create control effects only at those points of the control object, where it is needed, thus stabilizing the temperature field in a certain range $T_{\text {mid }}=T_{\text {det }}$ the system engage to a stable state. The process at the steady state: 


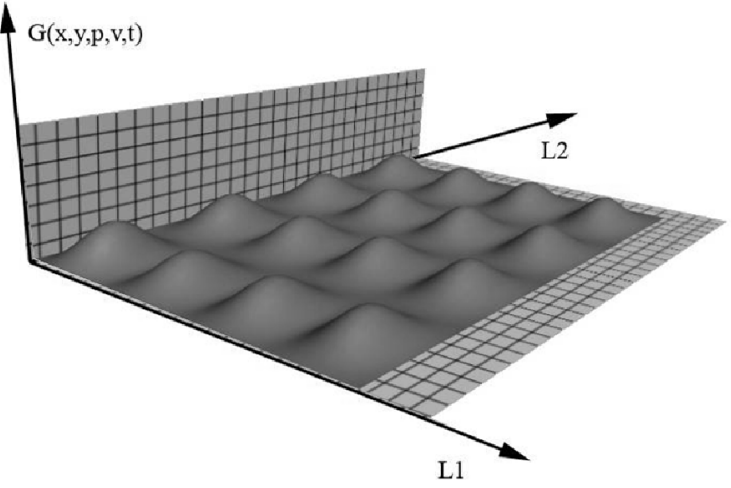

Figure 5. The system in a stable state

As it has been studied with a cylindrical object, let us investigate the distribution of heat through the plate, with the various characteristics of the control object.

According to the analysis of the conducted research it is clear that the processes in the two-dimensional and one-dimensional objects occur equally (Chernyshev, 2010; Chernyshev, et al., 2010; Chernyshev, 2008).

Namely, if we make a longitudinal or transverse section of the same one-dimensional or two-dimensional object, we obtain the same values of the temperature field. In order to confirm this statement let us analyze the three-dimensional control object and compare the obtained results. Spatially distributed model of thermal process control will be presented in the form of:

$$
\begin{gathered}
\frac{\partial Q(x, y, z, t)}{\partial t}-a^{2}\left[\frac{\partial^{2} Q(x, y, z, t)}{\partial x^{2}}+\frac{\partial^{2} Q(x, y, z, t)}{\partial y^{2}}+\frac{\partial^{2} Q(x, y, z, t)}{\partial z^{2}}\right]=f(x, y, z, t) \\
Q(x, y, z, 0)=Q_{0}(x, y, z) \\
Q(0, y, z, t)=q_{1}(y, z, t) ; \quad Q\left(l_{1}, y, z, t\right)=q_{2}(y, z, t) ; \quad Q(x, 0, z, t)=q_{3}(y, z, t) \\
Q\left(x, l_{2}, z, t\right)=q_{4}(x, z, t) ; \quad Q(x, y, 0, t)=q_{5}(x, y, t) ; \quad Q\left(x, y, l_{3}, t\right)=q_{6}(x, y, t) \\
0 \leq x \leq l_{1} ; \quad 0 \leq y \leq l_{2} ; 0 \leq z \leq l_{3} ; t \geq 0 ; a>0
\end{gathered}
$$

Let us investigate the temperature field according to the following system parameters: $l_{1}=10, l_{2}=10, l_{3}=10$, $a^{2}=0.01, \quad k=10, \quad d=9, \quad \tau=3, \quad x_{1}=y_{1}=z_{1}=p_{1}=v_{1}=\vartheta_{1}=1, \quad \mathrm{t}=1 . .500, \quad \xi_{i}, p_{1}, v_{1}, \vartheta_{1} \in\{1,2,3,4,5,6,7,8,9\}$. These parameters are identical to the two-dimensional and one-dimensional cases. The obtained result can be considered as adequate. However, because of the large dimension it is impossible to simulate the model in the programming media. This is due to the fact that the range of the temperature field values goes beyond the data set and can not be counted. Let us compare the results, obtained in Table 5.

Table 5. The Summary table of the values of the temperature processes simulation in one, two and three-dimensional control object

\begin{tabular}{lll}
\hline One-dimensional object & Two-dimensional object & Three-dimensional object \\
\hline 1.09330795587699 & 0.19038308199755 & 0.0830696784939504 \\
1.04499510571529 & 0.156279516336947 & 0.0617807374115696 \\
1 & 0.12828496613383 & 0.0459476861391161 \\
0.958058401181264 & 0.105305115613973 & 0.0341722994899598 \\
0.918928596838002 & 0.0864416751913374 & 0.0254146868004607 \\
0.882389406938066 & 0.0709572670455638 & 0.0189014586318749 \\
0.848238371316542 & 0.0582466007910039 & 0.014057428337303 \\
0.816290100849305 & 0.0478128124851264 & 0.0104548170227012 \\
0.786374777327054 & 0.0392480420606954 & 0.00777547616501912 \\
0.758336788421048 & 0.0322174899474342 & 0.0057827917467617 \\
0.732033485388861 & 0.0264463296514984 & 0.00430078874614273 \\
0.707334052308812 & 0.021708964701374 & 0.00319859068922997 \\
0.684118476666051 & 0.0178202100108361 & 0.00237886187885992 \\
\hline
\end{tabular}




\begin{tabular}{llc}
\hline 0.662276612051645 & 0.0146280529356706 & 0.00176921162740435 \\
0.641707324587187 & 0.0120077110515906 & 0.00131580139660855 \\
0.622317715459757 & 0.00985675437001555 & 0.000978590287617036 \\
0.60402241265265 & 0.00809110131759468 & 0.000727799007879672 \\
0.586742925593023 & 0.00664173196105313 & 0.000541280045973567 \\
0.570407057014391 & 0.00545198999628748 & 0.000402561813079011 \\
0.554948366855309 & 0.00447536803561486 & 0.000299394028202131 \\
0.540305683490524 & 0.00367368961935767 & 0.000222665889338851 \\
0.526422658021911 & 0.00301561688602492 & 0.000165601493699762 \\
0.513247357747662 & 0.00247542556544792 & 0.000123161454127618 \\
0.500731895283271 & 0.00203199940896687 & $9.15978681347563 \mathrm{e}-005$ \\
0.488832090130069 & 0.00166800474862777 & $6.81233386392012 \mathrm{e}-005$ \\
0.477507159779612 & 0.00136921291864911 & $5.06648174444833 \mathrm{e}-005$ \\
0.466719437707739 & 0.00112394405240028 & $3.76805332498149 \mathrm{e}-005$ \\
0.456434115853216 & 0.000922610512740643 & $2.80238370057525 \mathrm{e}-005$ \\
0.44661900939473 & 0.000757342108267506 & $2.08419407262195 \mathrm{e}-005$ \\
0.437244341838734 & 0.000621678445058328 & $1.55006073274719 \mathrm{e}-005$ \\
0.428282548611089 & 0.000510316387839917 & $1.15281408135958 \mathrm{e}-005$ \\
0.41970809750934 & 0.000418902758762284 & $8.57373055199954 \mathrm{e}-006$ \\
$* * * * * * * * * * * * * * * *$ & $* * * * * * * * * * * * * * * *)$ & $* * * * * * * * * * * * * * * * *$ \\
0.0286438707682219 & $4.71693549537191 \mathrm{e}-044$ & $1.02444796925336 \mathrm{e}-066$ \\
0.0285300366678357 & $3.87198479021778 \mathrm{e}-044$ & $7.61904369051661 \mathrm{e}-066$ \\
\hline
\end{tabular}

From the obtained results it is clear that the value of the temperature field, with the same initial data, decreases with accordance of the degree of the equation.

However, it should be noted that the conformities, observed within the considering of one-dimensional case, are identical to the conformities of two and three-dimensional objects.

For example, the first heating impulse has maximum amplitude and, in the course of time, to maintain the predetermined temperature value, short pulses at certain locations of the object are sufficient, which in its turn suggests the irrational use of solid heating elements, located throughout the whole spatial object area.

For instance, in tunnel kilns, for porcelain and brick firing as well as for the bakery oven the solid heating elements are used, which are arranged in the zones of heating over the entire area of the heated surface.

At the same time, the tests showed that to maintain the temperature of the product (brick, statue, bun, etc.) it is not necessary to use solid heaters, and the use of point impulse heating elements with relay control principle is sufficient.

\section{Discussion}

The actuality of the conducted research is defined by the complexity of the implementation of nonlinear control systems by the objects with distributed parameters. Controlled values of such systems depend not only on time but also on the distributed spatial region, occupied by the object. In this regard, the class of control effects expands essentially, primarily due to the possibility of their inclusion to the number of space-time controls, described by the functions of several variables - time and space coordinates.

Peculiarities of systems with distributed parameters require the creation of an apparatus for analysis and synthesis, based on non-traditional classical control theory of mathematical tools. There are various forms of model description of systems with distributed parameters: in the form of differential equations in partial derivative; structural representation of systems with distributed parameters, which is based on the fundamental solution of the boundary task; representation of distributed objects in the form of complex of transfer coefficients by their own vector of the operator object.

For the analysis of control objects described by nonlinear partial differential equations, the most commonly used approximation methods. However, it should be noted that to date, the approximation technique of distributed systems specially chosen centered system has not been developed, thus, in many problems of the process of approximation is unstable with respect to errors in intermediate calculations. Development of models for the systems under consideration and synthesis techniques have recently engaged in a large number of authors in mind unconditional urgency and great demand for technical solutions in practice. However, many of the end 
stages of modeling systems, suggesting further parametric synthesis, the use of which is connected with the solution of some problems. The proposed method compares favorably with the fact that it is brought to its logical conclusion - derived control algorithms.

The scientific value of this work is to develop the theoretical foundations of the analysis and synthesis of nonlinear distributed control systems.

The practical significance of the work lies in the fact that the developed method of calculating the installation location of the heating elements, depending on the temperature field, we can consider the installation of electric tunnel kilns conveyor type sectional impulse heaters. Analysis of the results of the developed software to stabilize the temperature fields shown:

1) The possibility of the achieving the desired temperature range of the kiln through the use of impulse heating elements.

2) The ability to stabilize the temperature field within the permissible range. The dependence of temperature on the length of the section is regarded.

\section{Conclusion}

The above presented methodology is considering a possibility of replacing of solid heating elements to the impulsive elements. Novelty and technical peculiarity of this paper is as follows:

1. Usage an innovative approach to the heating of hexagonal silicon carbide structures is an important task, since rods of this alloy are used for firing ceramics, bricks and other products.

2. This method is not designed solely for the hexagonal silicon carbide the structure, and has a general form which can be easily adapted to other alloys.

3. The offered method will reduce the final cost of the product by saving energy resources of a company

This technique, together with the hardware-software complex for the stabilization of the temperature field of conveyor type tunnel kilns will solve a wide range of tasks, required for modern industry (Tikhonov, \& Samara 1965; Chernyshev, 2010; Chernyshev, 2008).

1. Thus, the developed method can be generalized to the class of systems, for which there exists a fundamental solution (Green's function). At the same time, the increasing complexity of expression of the Green' function, naturally causes an increase in the cost of the computational process. However, if you compare the costs, which are now being spent on the low efficiency of the heating elements, the use of mathematical modeling to calculate the location of the heating elements is justified (Kolesnikov, et al. (2007); Zarembo, \& Kolesnikov (2006); Zarembo, et al. (2006); Zarembo, et al. (2004); Rapoport, et al. (2010); Pleshivtseva, et al. (2009); Rapoport (2006); Rapoport, \& Pleshivtseva (2006); Rapoport (1996); 1Ilyushin, et al. (2014); Chernishev (2009); Chernishev (2009); Chernishev (2010); Chernishev, et al. (2010); Chernishev (2008)).

It should also be noted that it would be useful to consider the choice of the parameters of the discretization of control effects for systems, boundary problem of which contains a non-zero boundary conditions. The fundamental solution (Green's function) of the boundary problem of such systems has the form that is different from the type, considered in the work. Also, the possibility of extending the working area of the object should be explored, that is an area within which, with a given accuracy, it becomes possible to achieve the desired value of the output function. But this is a subject for further researches.

\section{Acknowledgements}

It should be noted that persons, without whom these studies could not have been carried out. The first is Pershin Ivan Mitrofanovich (doctor of technical sciences, professor, actual member of the Academy of Natural Sciences, Honored Worker of Higher Professional Education of the Russian Federation), Kolesnikov Anatoly Arkadievich (Honored Worker of Science and Technology of the Russian Federation, doctor of technical sciences, professor, member of the Academy of Sciences and the Academy of electrical-technical sciences and Motion Control, Corresponding Member of the Russian Academy of Natural Sciences, Soros Professor (four) in the exact sciences field), and Chernyshev Alexander Borisovich (doctor of Technical Sciences, Professor), laid the foundation for the synthesis of distributed controllers on the basis of the Green's function.

\section{References}

Chernishev, A. (2008). The conditions of applicability of absolute stability criterion for distributed control systems. Almanac of modern science and education, 1(8), 214-215. 
Chernishev, A. (2009). Adaptation of absolute stability frequency criterion to systems with distributed parameters. Mechatronics, automatization, control, 7, 13-18.

Chernishev, A. (2009). Modified absolute stability criterion for nonlinear distributed systems. IHL News - North Caucasian region. Technical sciences, 3(151), 38-41.

Chernishev, A. (2010). Interpretation of absolute stability criterion for nonlinear distributed systems. Automatization and modern technologies, 2, 28-32.

Chernishev, A., Antonov, V., \& Shurakov, D. (2010). System of temperature field stabilization criterion in the process of heat utilization in contact welding. Scientific-technical news of S.Pt.SPI, 6(113), 151-155.

Ilyushin, Y. (2011). Designing of temperature field control system of tunnel kilns of conveyor type. Scientific-technical news of S.Pt.SPI, 3(126), 67-72.

Ilyushin, Y., Pervukhin, D., Afanasieva, O., Klavdiev, A., \& Kolesnichenko, S. (2014). Designing of Distributed Control System with Pulse Control. Middle-East Journal of Scientific Research, 21(3), 436-439. http://dx.doi.org/10.5829/idosi.mejsr.2014.21.03.21433

Kolesnikov, A. (2009). Nonlinear Oscillations Control. Energy Invariants. Journal of Computer and Systems Sciences International, 48(2), 185-198. http://dx.doi.org/10.1134/S1064230709020038

Kolesnikov, A., Zarembo, Y., \& Zarembo, V. (2007). Discharge of a Copper-Magnesium Galvanic Cell in the Presence of a Weak Electromagnetic Field. Russian Journal of Physical Chemistry A, 81(7), 1178-1180. http://dx.doi.org/10.1134/s003602440707031x

Kolesnikov, A., Zarembo, Y., Puchkov, L., \& Zarembo, V. (2007). Zinc Electrochemical Reduction on a Steel Cathode in a Weak Electromagnetic Field. Russian Journal of Physical Chemistry A, 81(10), 1715-1717. $\mathrm{http}: / / \mathrm{dx}$.doi.org/10.1134/s0036024407100330

Pershin, I. (2007). Analysis and synthesis of systems with distributed parameters. Pyatigorsk: RIA-KMV.

Pleshivtseva, Y., \& Rapoport, E. (2009). The Successive Parameterization Method of Control Actions in Boundary Value Optimal Control Problems For Distributed Parameter Systems. Journal of Computer and Systems Sciences International, 48(3), 351-362. http://dx.doi.org/10.1134/S1064230709030034

Rapoport, E. (1996). Alternance Properties of Optimal Solutions and Computational Algorithms in Problems of Semi-Infinite Optimization of Controlled Systems. Journal of Computer and Systems Sciences International, 35(4), 581-591.

Rapoport, E. (2006). Structural Parametric Synthesis of Automatic Control Systems with Distributed Parameters. Journal of Computer and Systems Sciences International, 45(4), 553-566. http://dx.doi.org/10.1134/S1064230706040071

Rapoport, E., \& Pleshivtseva, Y. (2010). Combined Optimization of Metal Hot Forming Line with Induction Pre-Heating. IEEE $26^{\text {th }}$ Convention of Electrical and Electronics Engineers, Israel, Eilat.

Rapoport, E., \& Pleshivtseva, Yu. (2006). Models and Methods of Semi-Infinite Optimization Inverse Heat-Conduction Problems. Heat Transfer Research, 37(3), 221-231.

Tikhonov, A., \& Samarsky, A. (1965). Equations of mathematical physics. Moscow: Science.

Zarembo, V., \& Kolesnikov, A. (2006). Background Resonant Acoustic Control of Heterophase Processes. Theoretical Foundations of Chemical Engineering, 40(5), 483-495. http://dx.doi.org/10.1134/s0040579506050058

Zarembo, V., Kolesnikov, A., \& Ivanov, E. (2006). Background Electromagnetic-Acoustic Control of Structural And Plastic Properties of Metals. Bulletin of the Russian Academy of Sciences: Physics, 70(8), 1239-1243.

Zarembo, V., Kiseleva, O., Kolesnikov, A., Burnos, N., \& Suvorov, K. (2004). Structuring of Inorganic Materials in Weak $\mathrm{Rf}$ Electromagnetic Fields. Inorganic Materials, 40(1), 86-91. http://dx.doi.org/10.1023/B:INMA.0000012184.66606.59

\section{Copyrights}

Copyright for this article is retained by the author(s), with first publication rights granted to the journal.

This is an open-access article distributed under the terms and conditions of the Creative Commons Attribution license (http://creativecommons.org/licenses/by/3.0/). 